\title{
Position Enhanced Mention Graph Attention Network for Dialogue Relation Extraction
}

\author{
Xinwei Long \\ Institute of Software Chinese Academy of Sciences \\ University of Chinese Academy of Sciences \\ Beijing, China \\ longxinwei19@mails.ucas.ac.cn
}

\author{
Shuzi Niu, Yucheng Li \\ Institute of Software Chinese Academy of Sciences \\ Beijing, China \\ \{shuzi,yucheng\}@iscas.ac.cn
}

\begin{abstract}
Dialogue Relation Extraction (DRE) is a new kind of relation extraction task from multi-turn dialogues. Different from the previous tasks, speaker specific relations are implicitly mixed together in both a local utterance window and a speaker context. To tackle both local and speaker dependency challenges, we explicitly construct a unified mention co-occurrence graph within a local utterance window or all utterances of a speaker from different entities. For each dialogue, a position enhanced graph attention network over this graph is proposed to obtain position aware mention representations in terms of both contexts. A gate function is utilized to help obtain a discriminative representation enough for each relation from original and position aware mention representations. For each entity pair in this dialogue, a pairwise attention mechanism is deployed to aggregate those discriminative mention representations as pair representation, which is fed into a standard multi-label classifier for relation label prediction. Experimental results on two benchmarks show the performance improvement of the proposed method is at least $1.6 \%$ and $3.2 \%$ compared with SOTA.
\end{abstract}

\section{CCS CONCEPTS}

- Information systems;

\section{KEYWORDS}

Dialogue Relation Extraction, Graph Neural Network, Attention Mechanism

\section{ACM Reference Format:}

Xinwei Long and Shuzi Niu, Yucheng Li. 2021. Position Enhanced Mention Graph Attention Network for Dialogue Relation Extraction. In Proceedings of the 44th International ACM SIGIR Conference on Research and Development in Information Retrieval (SIGIR '21), July 11-15, 2021, Virtual Event, Canada. ACM, New York, NY, USA, 5 pages. https://doi.org/10.1145/3404835.3463070

\section{INTRODUCTION}

Widely used in knowledge based applications, Relation Extraction (RE) attempts to predict relation labels between entities from a piece of text. Recent dialogue is treated as natural human-computer interaction way attracting more and more attentions. With dialogue

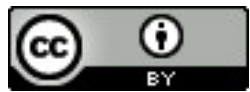

This work is licensed under a Creative Commons Attribution International 4.0 License. SIGIR '21, July 11-15, 2021, Virtual Event, Canada. (C) 2021 Copyright held by the owner/author(s). ACM ISBN 978-1-4503-8037-9/21/07.

https://doi.org/10.1145/3404835.3463070 related natural language processing technique more and more significant, Dialogue Relation Extraction (DRE) task emerges.

Dialogue Relation Extraction task puts emphasis on speaker relation extraction from multi-turn dialogue text. In fact, these speaker relations are implicitly mixed together in both a local utterance context and a speaker context, which leads to the local and speaker dependency challenges. We can tell the word meaning from its context, but the context omission is common in dialogue text.

Local Dependency Challenge. Different relations are implicitly coupled in a local utterance window, especially relations with the same head or tail entity, which requires more discriminative representations. For example in Fig. 1, (Speaker1, per:sibling, Speaker2) and (Speaker2, per:alias, Rose) are mixed in the first two utterances. The omitted context of Rose leads to an implicit relation, which makes it more difficult to decouple features of each mention for different relations.

Speaker Dependency Challenge. A speaker context is usually composed of several utterances which follow in interrupted order and scattered in the whole dialogue. Multiple relations are implicitly mentioned in a speaker context, which does not exist in previous tasks. Both (Speaker4, per:alias, Dad) and (Speaker2, per:alias, Rose) can be inferred from Speaker1's utterances. The second triplet helps differentiate roles of "Dad" mentions in different positions to predict the first triplet, which is ignored by most RE methods.

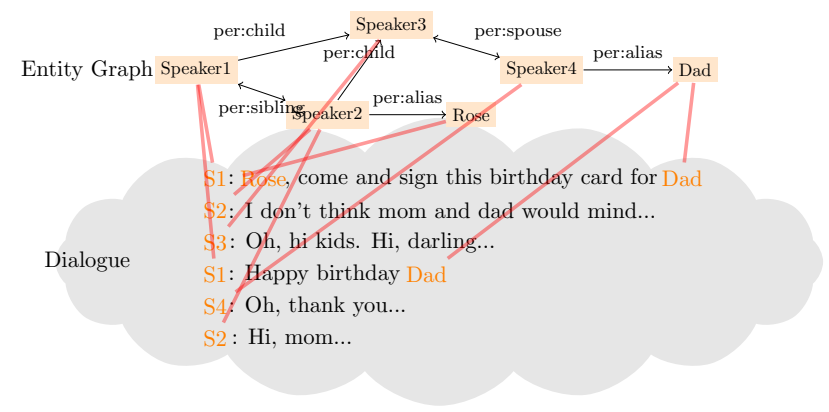

Figure 1: Dialogue example from DialogRE dataset.

To tackle both challenges in DRE, we propose a position enhanced mention graph attention network, referred to as PMGAN. The major contribution lies in the position enhanced mention graph attention module. We first design a mention co-occurrence graph within a local utterance or global speaker context for each dialogue to accommodate these mixed relations simultaneously. Then position enhanced graph attention mechanism is performed over this 
mention co-occurrence graph to obtain position aware mention representations. Next, a fusion gate function is used to balance position aware and original representations to derive a discriminative mention representation. Finally, a pairwise attention mechanism for each entity pair is adopted to aggregate discriminative mention representations as pair representations for relation classification.

We conduct comprehensive experiments on two benchmark datasets, DialogRE [11] and MPDD [2]. Our model achieves the 1.6\% and $3.2 \%$ improvement in terms of F1 on DialogRE and accuracy on MPDD than the state-of-the-art models respectively. Ablation studies show that each module plays an essential role.

\section{RELATED WORK}

Here we briefly review the recent progresses in traditional and dialogue relation extraction methods.

Early studies focus on predicting relations for given two entities within a sentence, ignoring the interaction cross the entities and failing to detect the overlapped relations. Recent work designs seq2seq tagging mechanism [14] or relation specific table-filling method [12] to improve the performance in overlapped scenarios. Moreover, some studies [13,15] shift their attention to detecting relations in long text, expanding their extraction scope from a sentence to an entire document. The task [10] requires aggregating information from local to global and performing reasoning over triples across a long distance. Typical studies are mainly graphbased approaches, which construct a document-level graph and deploy a graph neural network to learn the representations. Specifically, [15] proposes a global context-enhanced graph convolution network to capture rich global context features, while [13] introduces an entity-level graph and path-based reasoning mechanism to infer relations over documents.

Recently, [11] proposes a dialogue relation extraction dataset, named DialogRE, consisting of 1788 dialogues and 36 relations. Dialogue RE task also faces the challenges of overlapped relations and reasoning over triples, making it more intractable to tackle. HGAT [1] constructs a heterogeneous document graph to model the dialogues and GDPNet [9] proposes a latent multi-view latent graph to capture important features in a long text. SimpleRE [8] designs a relation refinement mechanism and reports the SOTA performance. The above approaches ignore the latent interaction between the different triples, which results in the bottlenecks to detect the overlapped triples and complicated inter-speaker relations.

\section{POSITION ENHANCED MENTION GRAPH ATTENTION NETWORK}

In this section, we first formalize the dialogue relation extraction task. To solve the relation perplexing problem in both utterance and speaker context, Position enhanced Mention Graph Attention Network (PMGAN) will be introduced in detail.

\subsection{Problem Formulation}

There are $N$ dialogues dealing with $M$ relations denoted as $R$ in dialogue relation extraction dataset $D=\left\{\left(U_{i}, S_{i}, G_{i}\right)\right\}_{i=1}^{N}$. For each $(U, S, G) \in D$, a dialogue $U$ is composed of $T$ utterances $U=$ $\left\{u_{1}, \ldots, u_{T}\right\}$ generated by $S$ speakers, and $G$ is the ground truth entity graph $G$. Each triplet $\left(e_{i}, R_{i j}, e_{j}\right) \in G$ means that there exist a set of relations $R_{i j} \subset R$ between $e_{i}$ and $e_{j}$. Each utterance $u_{t} \in U$ is naturally a word sequence from one or more speakers. Dialogue relation extraction task attempts to learn a binary relation classifier $P\left(r\right.$ holds $\left.\mid\left(e_{i}, e_{j}\right)\right)$ for each relation $r$ based on $D$.

\subsection{Overall Architecture}

Taking the concatenation of all utterances in a dialogue $U$ as input, pre-trained BERT outputs all word representations, from which we first obtain the initialized mention representations for each entity. To accommodate these mixed relations, we design a mention cooccurrence graph within a local utterance window or a speaker's utterances. To make these initialized mention representations position aware, we then propagate these representations through a position enhanced mention graph attention module over this graph shown in the right part of Fig. 2. We further combine the initialized representations and their position aware versions by fusion gate in the left part of Fig. 2 to make mention representations discriminative enough for relation recognition. Based on these discriminative mention representations, we employ pairwise attention mechanism between entities and obtain each entity pair representation for classification. The whole process mentioned above is depicted in Fig. 2.

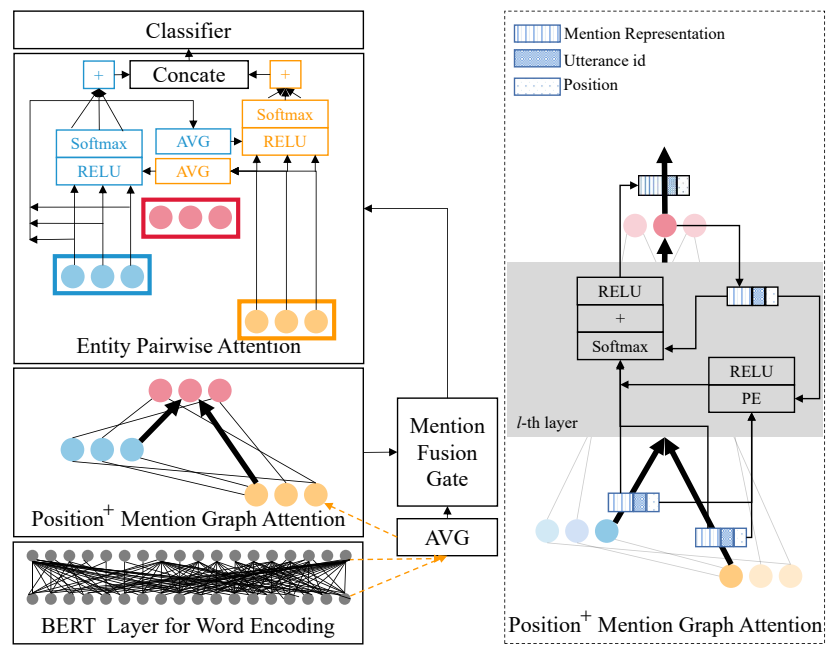

Figure 2: Overall architecture of our model.

3.2.1 Encoding Module. For each dialogue $U$ with $T$ utterance, we concatenate all the utterances as an input word sequence like " $[c l s],[s 1], w_{11}, w_{12}, \ldots,\left[s_{i}\right], \ldots, w_{i j}$, [sep]". [cls] and [sep] are the start and end word of the input, $s_{i}$ is added as a special token representing the speaker index of utterance $u_{i}$, and $w_{i j}$ is the $j$-th word in $u_{i}$. After encoding with pre-trained BERT, word representations I $\in \mathbb{R}^{n_{w} \times d}$ are obtained, where $n_{w}$ is the input sequence length and $d$ is the dimension of learned word representations.

For each entity $e \in V_{G}$, there are $n_{e}$ mentions $\mathcal{M}_{e}$ in dialogue $U, V_{G}$ is the entity set in ground truth entity graph $G$. For $e$ 's $j$-th mention $m_{j} \in \mathcal{M}_{e}$, it starts from $p_{j}^{s}$ and ends at $p_{j}^{e}$ in the input sequence and its representation is computed as the average 
word representations in Eq.(1). All mention representations for $U$ is denoted as $\mathbf{E} \in \mathbb{R}^{n_{m} \times d}$, where $n_{m}$ is the number of mentions in $U$.

$$
\mathbf{m}_{j}=\frac{1}{p_{j}^{e}-p_{j}^{s}+1} \sum_{k=p_{j}^{s}}^{p_{j}^{e}} \mathbf{I}_{k} .
$$

3.2.2 Position Enhanced Graph Attention Module. As mentioned before, each entity is corresponding to multiple mentions, which are scattered in the whole dialogue. Due to the semi-structured characteristic of the dialogue, relations among mentions are often coupled within a local utterance window or a speaker's utterances. Thus, we propose a position enhanced graph attention module to differentiate mentions from mixed relations with their position information and obtain position aware mention representations.

We first design a mention co-occurrence graph to include all possible entity relations. Specifically, each mention in $U$ is treated as a node. To accommodate these mixed relations, we define two kinds of co-occurrence edges between mentions. (1)Edge in a local context. If $e_{k_{1}}$ 's mention $m_{k_{1}, l_{1}}$ and $e_{k_{2}}$ 's mention $m_{k_{2}, l_{2}}$ are located in the same utterance window, i.e. $\left|\operatorname{uid}\left(m_{k_{1}, l_{1}}\right)-\operatorname{uid}\left(m_{k_{2}, l_{2}}\right)\right| \leq K$ and $k_{1} \neq k_{2}$, there is an edge between $m_{k_{1} . l_{1}}$ and $m_{k_{2}, l_{2}}$. uid $\left(m_{k_{2}, l_{2}}\right)$ is the utterance id of $m_{k_{2}, l_{2}}$. The window size $K$ is a hyper parameter fine-tuning during the training. (2)Edge in a speaker context. If mention $m_{k_{1}, l_{1}}$ and $m_{k_{2}, l_{2}}$ are generated by the same speaker and $k_{1} \neq k_{2}$, there is an edge between $m_{k_{1} . l_{1}}$ and $m_{k_{2}, l_{2}}$.

We propose a position enhanced graph attention mechanism over this graph to equip the mention representations with right distinctions for the following relation classification, such as mention positions. For each dialogue $U$, mention representations are initialized as $\mathbf{h}^{(0)}=\mathbf{E}$. At the $l$-th layer, we update the representation of $m_{i, j}$ based on position information and representations of its neighbors $\mathcal{N}_{i, j}$. For each neighbor $m_{k, t} \in \mathcal{N}_{i, j}$, its relative positions are calculated as $d_{u}\left(m_{i, j}, m_{k, t}\right)=\operatorname{uid}\left(m_{i, j}\right)-\operatorname{uid}\left(m_{k, t}\right)$ and $d_{p}\left(m_{i, j}, m_{k, t}\right)=p_{i, j}^{s}-p_{k, t}^{s}$ in terms of utterance ids and positions respectively. The relative position information is encoded as Eq.(2) by a non-linear function ReLU based on the basic sinusoid position embedding in [3, 7], denoted as $\mathrm{PE}(\cdot), \mathbf{W}_{r}$ and $\mathbf{b}_{r}$ are both parameters. The attention weight of $m_{i, j}$ to its neighbor $m_{k, t}$ computed as Eq.(3) and (4) to take the relative position embedding into consideration. Mention representation $\mathbf{h}_{i, j}^{(l)}$ for mention $m_{i, j}$ is updated based on its neighbor representations and position information as Eq.(5). After $L$ layers, we obtain the position aware mention representations $\mathbf{h}^{(L)}$.

$$
\begin{gathered}
\mathbf{R}_{i, j}^{k, t}=\operatorname{ReLU}\left(\mathbf{W}_{N}\left(\begin{array}{c}
\operatorname{PE}\left(d_{u}\left(m_{i, j}, m_{k, t}\right)\right) \\
\operatorname{PE}\left(d_{p}\left(m_{i, j}, m_{k, t}\right)\right)
\end{array}\right)+\mathbf{b}_{N}\right) \\
s_{i, j}^{k, t}=\mathbf{h}_{i, j}^{(l-1)} \mathbf{W}_{s}^{(l)}\left(\mathbf{h}_{k, t}^{(l)}+\mathbf{R}_{i, j}^{k, t}\right) \\
a_{i, j}^{k, t}=\frac{\exp \left(s_{i, j}^{k, t}\right)}{\sum_{m_{o, q} \in \mathcal{N}_{i, j}} \exp \left(s_{i, k}^{o, q}\right)} \\
\mathbf{h}_{i, j}^{(l+1)}=\operatorname{ReLU}\left(\mathbf{W}_{p}^{(l)} \mathbf{h}_{i, j}^{(l)}+\sum_{m_{o, q} \in \mathcal{N}_{i, j}} a_{i, j}^{o, q} \mathbf{W}_{a}^{(l)} \mathbf{h}_{o, q}^{(l)}+\mathbf{b}_{p}^{(l)}\right)
\end{gathered}
$$

3.2.3 Mention Fusion Gate. To retain inherent features in mention $m_{i, j}$ from the basic BERT encoder and alleviate noises from other mentions, we deploy a fusion gate mechanism to make trade offs between the original input representation $\mathbf{h}_{i, j}^{(0)}$ and the output representation of the above module $\mathbf{h}_{i, j}^{(L)}$. Similar to [6], we design heuristic features, such as subtraction and element-wise multiplication as Eq.(6) to capture similar and different features between them for gate $\beta_{i, j}$, $\odot$ denotes element-wise multiplication. Gate $\beta_{i, j}$ is used to adjust the weights of both representations and we obtain the mention representation $\mathbf{h}_{i, j}^{*}$ through Eq.(8).

$$
\begin{aligned}
\mathbf{B}_{i, j}^{*} & =\left(\mathbf{h}_{i, j}^{(0)} ; \mathbf{h}_{i, j}^{(L)} ; \mathbf{h}_{i, j}^{(0)}-\mathbf{h}_{i, j}^{(L)} ; \mathbf{h}_{i, j}^{(0)} \odot \mathbf{h}_{i, j}^{(L)}\right) \\
\beta_{i, j} & =\sigma\left(\mathbf{W}_{b} \mathbf{B}_{i, j}^{*}+\mathbf{b}_{b}\right) \\
\mathbf{h}_{i, j}^{*} & =\beta_{i, j} \odot \mathbf{h}_{i, j}^{(0)}+\left(1-\beta_{i, j}\right) \odot \mathbf{h}_{i, j}^{(L)}
\end{aligned}
$$

3.2.4 Entity Pairwise Attention. To learn discriminative entity pair representations for relations, we employ a pairwise attention mechanism. Given an entity pair $\left(e_{i}, e_{k}\right)$ in dialogue $U$, we obtain their coarse representations by averaging mention representations as

$$
\overline{\mathbf{e}}_{i}=\frac{1}{n_{e_{i}}} \sum_{j} \mathbf{h}_{i, j}^{*}, \overline{\mathbf{e}}_{k}=\frac{1}{n_{e_{k}}} \sum_{t} \mathbf{h}_{k, t}^{*} .
$$

For entity pair $\left(e_{i}, e_{k}\right)$, we obtain $e_{i}$ 's fine representation $\widetilde{\mathbf{e}}_{i}$ based on how $\overline{\mathbf{e}}_{k}$ attends to all $e_{i}$ 's mentions as Eq.(10) and obtain $e_{k}$ 's fine representation $\widetilde{\mathbf{e}}_{k}$ based on how $\overline{\mathbf{e}}_{i}$ attends to all $e_{k}$ 's mentions similarly. This attention mechanism learns one entity representation from the other entity, referred to as pairwise attention. Through this pairwise attention, we learn which "Dad" from Speaker1 is more important for per:alias relation prediction in Fig. 1.

$$
\begin{aligned}
& s_{i, j}^{k}=\operatorname{ReLU}\left(\overline{\mathbf{e}}_{k} \mathbf{W}_{m_{1}} \mathbf{h}_{i, j}^{*}+\mathbf{W}_{m_{2}} \mathbf{h}_{i, j}^{*}+b_{m}\right) \\
& \widetilde{\mathbf{e}}_{i}=\sum_{j} a_{i, j}^{k} \cdot \mathbf{h}_{i, j}^{*}, \text { where } a_{i, j}^{k}=\frac{\exp \left(s_{i, j}^{k}\right)}{\sum_{j^{\prime}} \exp \left(s_{i, j^{\prime}}^{k}\right)}
\end{aligned}
$$

\subsection{Inference and Learning}

With the concatenation of fine representations as the entity pair representation $\left(\widetilde{\mathbf{e}}_{i}, \widetilde{\mathbf{e}}_{k}\right)$, we use a feed forward neural network layer followed by a binary sigmoid classifier for each relation $r$ to decide the probability that relation $r$ holds in given $\left(e_{i}, e_{k}\right)$ as Eq.(11). The binary cross entropy loss is used to learn model parameters.

$$
P\left(r \mid e_{i}, e_{k}\right)=\sigma\left(\mathbf{W}_{r} \operatorname{ReLU}\left(\mathbf{W}_{e}\left(\widetilde{\mathbf{e}}_{i}, \widetilde{\mathbf{e}}_{k}\right)+\mathbf{b}_{e}\right)+b_{r}\right)
$$

\section{EXPERIMENTS}

We investigate the effectiveness of PMGAN on two benchmark datasets and the roles of its key components.

\subsection{Experimental Settings}

Datasets and Metrics. (1) DialogRE [11]. We follow the standard settings of the original paper, and employ F1 score as the evaluation measure. (2) MPDD [2]. Here MPDD ${ }^{1}$ originated from the finegrained relation classification sub-task in [2]. To avoid information

\footnotetext{
${ }^{1}$ Since the resource paper does not provide a standard split of the dataset, we randomly divide the training, validation, and test set, with 1482 , 400, and 400 dialogues, respectively. Source codes and data are available at https://github.com/xinwei96/PMGAN_codes.
} 
leakage, we anonymizes speaker names and filter out the duplicate instances following the settings of DialogRE. Accuracy is used as the metric in line with the original paper.

Implementation Details. We adopt BERT-base architecture [4] with the fine-tuning rate of $2 \mathrm{e}-5$ for word encoding. We use BertTokenizer to split each input sequence into sub-word pieces. For those tokenized sequences longer than 512, we split them into two overlapped sub-sequences. We implement our model with Pytorch ${ }^{2}$, and train our model with AdamW optimizer [5] with learning rate of $5 \mathrm{e}-4$ and batch size of 4 . We set the window size $K$ to 5 and the number of mention graph attention layers $L$ is 2 . All experiments are conducted on a sever with two Geforce GTX 1080Ti GPUs.

Baseline Settings. The baseline methods include the following three kinds. (1) Benchmark models offered by dataset DialogRE: BERT and BERTs [11]; (2) Advanced document-level RE models: GCGCN [15] and GAIN [13]; (3) State-of-the-art approaches in the DialogRE challenge: HGAT [1], GDPNet [9], and SimpleRE [8] Except that HGAT [1] encodes tokens with LSTM, other baselines deploy the BERT-base model as the encoder.

\subsection{Performance Analysis}

All BERT based models in Tab. $1^{3}$ significantly outperform the LSTM based model HGAT [1]. HGAT utilizes a heterogeneous graph attention network to derive entity representations, and also takes the speaker information into consideration. But it cannot achieve as good performance as BERT based methods, the simple method just with BERT as the word encoder. This indicates the early good word encoder is more competitive than the following complex network structure. So we adopt a pre-trained BERT layer as word encoder owing to BERT's superior representation learning ability.

Table 1: Performance Comparison on DialogRE and MPDD.

\begin{tabular}{c|c|c|c|c}
\hline & \multicolumn{2}{|c|}{ F1-DialogRE } & \multicolumn{2}{c}{ Acc-MPDD } \\
\cline { 2 - 5 } & Dev & Test & Dev & Test \\
\hline BERT [4] & 60.6 & 58.5 & $31.0^{*}$ & $31.6^{*}$ \\
BERT [11] & 63.0 & 61.2 & $37.2^{*}$ & $36.9^{*}$ \\
\hline HGAT [1] & 57.7 & 56.1 & - & - \\
GDPNet [9] & 67.1 & 64.9 & - & - \\
SimpleRE [8] & - & 66.7 & $36.9^{*}$ & $37.9^{*}$ \\
\hline GCGCN [15] & $66.9^{*}$ & $67.6^{*}$ & $42.5^{*}$ & $39.1^{*}$ \\
GAIN [13] & $69.8^{*}$ & $69.0^{*}$ & $42.2^{*}$ & $43.6^{*}$ \\
\hline Our Model & $\mathbf{7 0 . 3}$ & $\mathbf{7 0 . 6}$ & $\mathbf{4 7 . 2}$ & $\mathbf{4 6 . 8}$ \\
\hline
\end{tabular}

Advanced document-level RE methods perform even better than state-of-the-art dialogue RE models in Tab. 1. Most of them are graph neural network based RE methods and various graph construction methods lead to different directions. The major reason lies in that dialogue RE models ignore the complex relation perplexing phenomenon, which is supposed to be significant for most document-level RE methods. That's why we introduce position information to mention graph attention module to obtain discriminative mention representations.

Our method outperforms the best state-of-the-art baseline GAIN about $1.6 \%$ and $3.2 \%$ in terms of F1 on DialogRE and accuracy

\footnotetext{
${ }^{2}$ https://pytorch.org/

${ }^{3} *$ represents the results produced by running author released codes.
}

on MPDD respectively in Tab. 1. Graph neural networks (GNN) help learn smoothed node representations among neighbors, which will prompt the performance of entity pairs with a ground truth relation and do harm to other entity pairs. Hence in our method a fusion gate is introduced to alleviate this problem and a pairwise attention mechanism is further used to derive relation specific representations. Both modules contribute to the performance gain.

\subsection{Ablation Studies}

We conduct ablation studies of three major modules to explore the necessity of each module of PMGAN. Some details of position enhanced graph attention module are also considered, i.e. replace co-occurrence graph with Fully connected graph and remove the relative position encoding unit. Results are demonstrated in Tab 2.

Table 2: Ablation studies on DialogRE.

\begin{tabular}{l|ccc}
\hline Model & $\mathrm{P}$ & $\mathrm{R}$ & $\mathrm{F} 1$ \\
\hline Full Model & 73.4 & 68.0 & 70.6 \\
\hline w.o. Position $^{+}$Graph Attention Network & 72.2 & 63.9 & 67.8 \\
w.o. Fusion Gate & 69.6 & 67.7 & 68.6 \\
w.o. Pairwise Attention & 72.0 & 64.0 & 67.8 \\
\hline Full Connection Mention Graph & 72.6 & 67.0 & 69.6 \\
w.o. Relative Position Encoding & 71.1 & 67.1 & 69.0 \\
\hline
\end{tabular}

The largest F1 improvement is achieved by adding position enhanced GAN or pairwise attention. This performance improvement mainly comes from the recall improvement, which is the direct effect of the smoothed representations through graph neural network in terms of position enhanced GAN. From pairwise attention over an entity pair, the semantic expansion from each other entity will help locate actual mentions as more as possible. In this sense, the recall improvement is achieved and so is F1. The addition of fusion gate module brings about $2.0 \% \mathrm{~F} 1$ improvement. Generally, three components are all necessary for the performance gain in Tab. 1.

Replacing the mention co-occurrence graph with fully connected graph, the performance decreases by $1 \%$ in terms of F1. Removing relative position encoding unit, F1 score drops by $1.6 \%$ compared with the full model. These comparison results suggest that position encoding, utterance, and speaker-related information play a necessary part in dialogue RE task. Feature propagation over the mention graph help alleviate noises from the unrelated mentions.

\section{CONCLUSION}

To tackle local and speaker dependency challenges, we propose a position enhanced mention graph attention network to obtain position aware mention representations and use a pairwise attention mechanism to derive entity pair specific representations for relation classification. Experimental results on benchmark datasets demonstrate a significant improvement compared with SOTA. Each module plays an essential role in performance improvement through ablation studies. In the future, we will try to integrate symbolic knowledge and logical reasoning into dialogue RE tasks.

\section{ACKNOWLEDGMENTS}

This research work was funded by the National Natural Science Foundation of China under Grant No. 62072447. 


\section{REFERENCES}

[1] Hui Chen, Pengfei Hong, Wei Han, Navonil Majumder, and Soujanya Poria 2020. Dialogue Relation Extraction with Document-level Heterogeneous Graph Attention Networks. CoRR abs/2009.05092 (2020). arXiv:2009.05092

[2] Yi-Ting Chen, Hen-Hsen Huang, and Hsin-Hsi Chen. 2020. MPDD: A Multi-Party Dialogue Dataset for Analysis of Emotions and Interpersonal Relationships. In Proceedings of The LREC. 610-614.

[3] Zihang Dai, Zhilin Yang, Yiming Yang, Jaime G. Carbonell, Quoc Viet Le, and Ruslan Salakhutdinov. 2019. Transformer-XL: Attentive Language Models beyond a Fixed-Length Context. In Proceedings of the 57th Conference of the Association for Computational Linguistics, ACL 2019, Florence, Italy, fuly 28-August 2, 2019. 2978-2988.

[4] Jacob Devlin, Ming-Wei Chang, Kenton Lee, and Kristina Toutanova. 2019. BERT: Pre-training of Deep Bidirectional Transformers for Language Understanding. In NAACL-HLT. 4171-4186.

[5] Ilya Loshchilov and Frank Hutter. 2019. Decoupled Weight Decay Regularization. In 7th International Conference on Learning Representations, ICLR 2019, New Orleans, LA, USA, May 6-9, 2019. OpenReview.net.

[6] Lili Mou, Rui Men, Ge Li, Yan Xu, Lu Zhang, Rui Yan, and Zhi Jin. 2016. Natural Language Inference by Tree-Based Convolution and Heuristic Matching. In Proceedings of the ACL. 130-136.

[7] Ashish Vaswani, Noam Shazeer, Niki Parmar, Jakob Uszkoreit, Llion Jones, Aidan N. Gomez, Lukasz Kaiser, and Illia Polosukhin. 2017. Attention is All you Need. In Advances in Neural Information Processing Systems 30: Annual Conference on Neural Information Processing Systems 2017, December 4-9, 2017, Long Beach, CA, USA. 5998-6008.
[8] Fuzhao Xue, Aixin Sun, Hao Zhang, and Eng Siong Chng. 2020. An Embarrassingly Simple Model for Dialogue Relation Extraction. CoRR (2020). arXiv:2012.13873

[9] Fuzhao Xue, Aixin Sun, Hao Zhang, and Eng Siong Chng. 2021. GDPNet: Refining Latent Multi-View Graph for Relation Extraction. In The Thirty-Fifth AAAI Conference on Artificial Intelligence AAAI 2021.

[10] Yuan Yao, Deming Ye, Peng Li, Xu Han, Yankai Lin, Zhenghao Liu, Zhiyuan Liu, and Maosong Sun. 2019. DocRED: A Large-Scale Document-Level Relation Extraction Dataset. In ACL. 764-777.

[11] Dian Yu, Kai Sun, Claire Cardie, and Dong Yu. 2020. Dialogue-Based Relation Extraction. In Proceedings of the 58th Annual Meeting of the ACL. 4927-4940.

[12] Yue Yuan, Xiaofei Zhou, Shirui Pan, Qiannan Zhu, Zeliang Song, and Li Guo. 2020. A Relation-Specific Attention Network for Joint Entity and Relation Extraction. In Proceedings of the Twenty-Ninth International foint Conference on Artificial Intelligence, IfCAI 2020, Christian Bessiere (Ed.). ijcai.org, 4054-4060. https: //doi.org/10.24963/ijcai.2020/561

[13] Shuang Zeng, Runxin Xu, Baobao Chang, and Lei Li. 2020. Double Graph Based Reasoning for Document-level Relation Extraction. In Proceedings of the 2020 Conference on EMNLP. 1630-1640.

[14] Xiangrong Zeng, Daojian Zeng, Shizhu He, Kang Liu, and Jun Zhao. 2018. Extracting Relational Facts by an End-to-End Neural Model with Copy Mechanism. In Proceedings of the 56th Annual Meeting of the ACL. 506-514.

[15] Huiwei Zhou, Yibin Xu, Weihong Yao, Zhe Liu, Chengkun Lang, and Haibin Jiang. 2020. Global Context-enhanced Graph Convolutional Networks for Documentlevel Relation Extraction. In Proceedings of the 28th International Conference on Computational Linguistics, COLING 2020, Barcelona, Spain (Online), December 8-13, 2020. 5259-5270. 